\title{
On the Security of Diffie-Hellman Bits
}

\author{
Maria Isabel González Vasco and Igor E. Shparlinski
}

\begin{abstract}
Boneh and Venkatesan have recently proposed a polynomial time algorithm for recovering a "hidden" element $\alpha$ of a finite field $\mathbb{F}_{p}$ of $p$ elements from rather short strings of the most significant bits of the remainder modulo $p$ of $\alpha t$ for several values of $t$ selected uniformly at random from $\mathbb{F}_{p}^{*}$. We use some recent bounds of exponential sums to generalize this algorithm to the case when $t$ is selected from a quite small subgroup of $\mathbb{F}_{p}^{*}$. Namely, our results apply to subgroups of size at least $p^{1 / 3+\varepsilon}$ for all primes $p$ and to subgroups of size at least $p^{\varepsilon}$ for almost all primes $p$, for any fixed $\varepsilon>0$. We also use this generalization to improve (and correct) one of the statements of the aforementioned work about the computational security of the most significant bits of the Diffie-Hellman key.
\end{abstract}

\section{Introduction}

Let $p$ be an $n$-bit prime and let $g \in \mathbb{F}_{p}$ be an element of multiplicative order $T$, where $\mathbb{F}_{p}$ is the finite field of $p$ elements.

For integers $s$ and $m \geq 1$ we denote by $(s \mathrm{rem} m)$ the remainder of $s$ on division by $m$. We also use $\log z$ to denote the binary logarithm of $z>0$.

In the case of $T=p-1$, that is, when $g$ is a primitive root, Boneh and Venkatesan [2] have proposed a method of recovering a "hidden" element $\alpha \in \mathbb{F}_{p}$ from about $n^{1 / 2}$ most significant bits of $\left(\alpha g^{x_{i}} \operatorname{rem} p\right), i=1, \ldots, d$, for $d=\left\lceil 2 n^{1 / 2}\right\rceil$ integers $x_{1}, \ldots, x_{d}$, chosen uniformly and independently at random in the interval $[0, p-2]$. This result has been applied to proving security of reasonably small portions of bits of private keys of several cryptosystems. In particular, in Theorem 2 of [2] the security of the $\left\lceil n^{1 / 2}\right\rceil+\lceil\log n\rceil$ most significant bits of the private key $\left(g^{a b} \operatorname{rem} p\right)$ of the Diffie-Hellman cryptosystem with public keys $\left(g^{a} \operatorname{rem} p\right)$ and $\left(g^{b}\right.$ rem $\left.p\right)$ with $a, b \in[0, p-2]$ is considered.

Namely, a method has been given to recover, in polynomial time, the DiffieHellman key $\left(g^{a b} \operatorname{rem} p\right)$ from $\left(g^{a} \operatorname{rem} p\right)$ and $\left(g^{b}\right.$ rem $\left.p\right)$, using an oracle which gives only the $\left\lceil n^{1 / 2}\right\rceil+\lceil\log n\rceil$ most significant bits of the Diffie-Hellman key.

Unfortunately the proof of Theorem 2 in [2] is not quite correct. Indeed, in order to apply Theorem 1 of that paper to $h=g^{b}$ this element must be a primitive root of $\mathbb{F}_{p}$. Thus the proof of Theorem 2 of [2] is valid only if $\operatorname{gcd}(b, p-1)=1$ (of course the same result holds in the case $\operatorname{gcd}(a, p-1)=1$ as well). However, even in 
the most favourable case when $l=(p-1) / 2$ is prime, only $75 \%$ of pairs $(a, b)$ satisfy this condition. Certainly breaking a cryptosystem in $75 \%$ of the cases is already bad enough (even in $0.75 \%$ is) but unfortunately for the attacker (using the above oracle), these weak cases can easily be described and avoided by the communicating parties. The proof of Theorem 3 of [2] suffers from a similar problem.

Here we use new bounds of exponential sums from [7] to extend some results of [2] to the case of elements $g$ of arbitrary multiplicative order $T$, provided that $T \geq p^{1 / 3+\varepsilon}$. This allows us to prove that the statement of Theorem 2 of [2] holds for all pairs $(a, b)$. We also prove that for almost all primes $p$ similar results hold already for $T \geq p^{\varepsilon}$.

A survey of similar results for other functions of cryptographic interest has recently been given in [5].

Throughout the paper the implied constants in symbols ' $O$ ' may occasionally, where obvious, depend on the small positive parameter $\varepsilon$ and are absolute otherwise; they all are effective and can be explicitly evaluated.

\section{Distribution of $g^{x}$ Modulo $p$}

For integers $\lambda, r$ and $h$ let us denote by $N_{\lambda, g, p}(r, h)$ the number of $x \in[0, T-1]$ for which $\left(\lambda g^{x} \operatorname{rem} p\right) \in[r+1, r+h]$.

We need the following asymptotic formula which shows that $N_{\lambda, g, p}(r, h)$ is close to its expected value $T h / p$, provided that $T$ is of larger order than $p^{1 / 3}$.

Lemma 2.1. For any $\varepsilon>0$ there exists $\delta>0$ such that for any element $g \in \mathbb{F}_{p}$ of multiplicative order $T \geq p^{1 / 3+\varepsilon}$ the bound

$$
\max _{0 \leq r, h \leq p-1} \max _{\operatorname{gcd}(\lambda, p)=1}\left|N_{\lambda, g, p}(r, h)-\frac{T h}{p}\right|=O\left(T^{1-\delta}\right)
$$

holds.

Proof. We remark that $N_{\lambda, g, p}(r, h)$ is the number of solutions $x \in\{0, \ldots, T-1\}$ of the congruence

$$
\lambda g^{x} \equiv y \quad(\bmod p), \quad y=r+1, \ldots, r+h .
$$

Using the identity (see Exercise 11.a in Chapter 3 of [17])

we obtain

$$
\sum_{c=0}^{p-1} \exp (2 \pi i c u / p)=\left\{\begin{array}{lll}
0, & \text { if } u \neq \equiv 0 & (\bmod p) \\
p, & \text { if } u \equiv 0 & (\bmod p)
\end{array}\right.
$$

$$
\begin{aligned}
N_{\lambda, g, p}(r, h) & =\frac{1}{p} \sum_{x=0}^{T-1} \sum_{y=r+1}^{r+h} \sum_{c=0}^{p-1} \exp \left(2 \pi i c\left(\lambda g^{x}-y\right) / p\right) \\
& =\frac{1}{p} \sum_{c=0}^{p-1} \sum_{x=0}^{T-1} \exp \left(2 \pi i c \lambda g^{x} / p\right) \sum_{y=r+1}^{r+h} \exp (-2 \pi i c y / p) .
\end{aligned}
$$


Separating the term $T h / p$ corresponding to $c=0$ we obtain

$$
\begin{aligned}
\left|N_{\lambda, g, p}(r, h)-\frac{T h}{p}\right| & \leq \frac{1}{p} \sum_{c=1}^{p-1}\left|\sum_{x=0}^{T-1} \exp \left(2 \pi i c \lambda g^{x} / p\right)\right|\left|\sum_{y=r+1}^{r+h} \exp (-2 \pi i c y / p)\right| \\
& =\frac{1}{p} \sum_{c=1}^{p-1}\left|\sum_{x=0}^{T-1} \exp \left(2 \pi i c \lambda g^{x} / p\right)\right|\left|\sum_{y=r+1}^{r+h} \exp (2 \pi i c y / p)\right|
\end{aligned}
$$

We estimate the sum over $x$ by using the bound

$$
\max _{\operatorname{gcd}(c, p)=1}\left|\sum_{x=0}^{T-1} \exp \left(2 \pi i c g^{x} / p\right)\right|=O(B(T, p)),
$$

where

$$
B(T, p)= \begin{cases}p^{1 / 2}, & \text { if } T \geq p^{2 / 3} \\ p^{1 / 4} T^{3 / 8}, & \text { if } p^{2 / 3}>T \geq p^{1 / 2} \\ p^{1 / 8} T^{5 / 8}, & \text { if } p^{1 / 2}>T \geq p^{1 / 3}\end{cases}
$$

which is essentially Theorem 3.4 of [7]. Using the estimate

$$
\max _{0 \leq r, h \leq p-1} \sum_{c=1}^{p-1}\left|\sum_{y=r+1}^{r+h} \exp (2 \pi i c y / p)\right|=O(p \log p),
$$

see Exercise 11.c in Chapter 3 of [17], we obtain

$$
\max _{0 \leq r, h \leq p-1}\left|N_{\lambda, g, p}(r, h)-\frac{T h}{p}\right|=O(B(T, p) \log p) .
$$

It is easy to see that for any $\varepsilon>0$ there exists $\delta>0$ such that $B(T, p)=O\left(T^{1-2 \delta}\right)$ for $T \geq p^{1 / 3+\varepsilon}$ and the result follows.

In the next statement we show that for almost all primes the lower bound $T \geq p^{1 / 3+\varepsilon}$ can be brought down to $T \geq p^{\varepsilon}$.

Lemma 2.2. Let $Q$ be a sufficiently large integer. For any $\varepsilon>0$ there exists $\delta>0$ such that for all primes $p \in[Q, 2 Q]$, except at most $Q^{5 / 6+\varepsilon}$ of them, and any element $g \in \mathbb{F}_{p}$ of multiplicative order $T \geq p^{\varepsilon}$ the bound

$$
\max _{0 \leq r, h \leq p-1} \max _{\operatorname{gcd}(\lambda, p)=1}\left|N_{\lambda, g, p}(r, h)-\frac{T h}{p}\right|=O\left(T^{1-\delta}\right)
$$

holds.

Proof. The proof is analogous to the proof of Lemma 2.1 using in this case Theorem 5.5 of [7] instead of (1) and (2). For each prime $p \equiv 1(\bmod T)$ we fix an element $g_{p, T}$ of multiplicative order $T$. Then Theorem 5.5 of [7] claims that for 
any $U>1$ and any integer $\nu \geq 2$, for all primes $p \equiv 1(\bmod T)$ except at most $O(U / \log U)$ of them, the bound

$$
\max _{\operatorname{gcd}(c, p)=1}\left|\sum_{x=0}^{T-1} \exp \left(2 \pi i c g_{p, T}^{x} / p\right)\right|=O\left(T p^{1 / 2 \nu^{2}}\left(T^{-1 / \nu}+U^{-1 / \nu^{2}}\right)\right),
$$

holds. We remark that the value of the above exponential sum does not depend on the particular choice of the element $g_{p, T}$.

Taking

$$
\nu=\left\lfloor\frac{1}{\varepsilon}\right\rfloor+1 \quad \text { and } \quad U=Q^{1 / 2+\varepsilon / 2},
$$

after simple computation we obtain that there exists some $\delta>0$, depending only on $\varepsilon$, such that for any fixed $T \geq Q^{\varepsilon / 2}$ the bound

$$
\max _{\operatorname{gcd}(c, p)=1}\left|\sum_{x=0}^{T-1} \exp \left(2 \pi i c g_{p, T}^{x} / p\right)\right|=O\left(T^{1-2 \delta}\right),
$$

holds for all except $O\left(Q^{1 / 2+\varepsilon / 2}\right)$ primes $p \equiv 1(\bmod T)$ in the interval $p \in[Q, 2 Q]$. As it follows from (1) and (2), a similar bound also holds for $T \geq Q^{1 / 3+\varepsilon / 2}$. So the total number of exceptional primes $p$ for which (3) does not hold for at least one $T \geq p^{\varepsilon}>Q^{\varepsilon / 2}$ is $O\left(Q^{5 / 6+\varepsilon}\right)$.

Using the bound (3) in the same way as we have used (1) and (2) in the proof of Lemma 2.1 we derive the desired result.

Certainly in both Lemma 1 and Lemma 3 the dependence of $\delta$ on $\varepsilon$ can be made explicit (as a linear function of $\varepsilon$ ).

\section{Lattices}

As in [2], our results rely on rounding techniques in lattices. We therefore review a few related results and definitions. vectors

Let $\left\{\mathbf{b}_{1}, \ldots, \mathbf{b}_{s}\right\}$ be a set of linearly independent vectors in $\mathbb{R}^{s}$. The set of

$$
L=\left\{\mathbf{z}: \mathbf{z}=\sum_{i=1}^{s} t_{i} \mathbf{b}_{i}, \quad t_{1}, \ldots, t_{s} \in \mathbb{Z}\right\}
$$

is called an $s$-dimensional full rank lattice. The set $\left\{\mathbf{b}_{1}, \ldots, \mathbf{b}_{s}\right\}$ is called the basis of $L$.

In [1] Babai describes a polynomial time algorithm which, for given a lattice $L$ and a vector $\mathbf{r}=\left(r_{1}, \ldots, r_{s}\right) \in \mathbb{R}^{s}$, finds a lattice vector $\mathbf{v}=\left(v_{1}, \ldots, v_{s}\right)$ satisfying the inequality

$$
\left(\sum_{i=1}^{s}\left(v_{i}-r_{i}\right)^{2}\right)^{1 / 2} \leq 2^{s / 4} \min \left\{\left(\sum_{i=1}^{s}\left(z_{i}-r_{i}\right)^{2}\right)^{1 / 2}, \quad \mathbf{z}=\left(z_{1}, \ldots, z_{s}\right) \in L\right\}
$$


That is, a given vector can be rounded in polynomial time to an approximately closest vector in a given lattice. The above algorithm uses the lattice basis reduction algorithm of Lenstra, Lenstra and Lovász [9], see also [14] for some more recent and stronger results.

For integers $x_{1}, \ldots, x_{d}$, selected in the interval $[0, T-1]$, we denote by $L_{g, p}\left(x_{1}, \ldots, x_{d}\right)$ the $d+1$-dimensional lattice generated by the rows of the following $(d+1) \times(d+1)$-matrix

$$
\left(\begin{array}{llllll}
p & 0 & 0 & \ldots & 0 & 0 \\
0 & p & 0 & \ldots & 0 & 0 \\
& \vdots & & & & \vdots \\
0 & 0 & 0 & \ldots & p & 0 \\
t_{1} & t_{2} & t_{3} & \ldots & t_{d} & 1 / p
\end{array}\right)
$$

where $t_{i}=\left(g^{x_{i}} \operatorname{rem} p\right), i=1, \ldots, d$.

The following result is a generalization of Theorem 5 of [2] (which corresponds to the case $T=p-1)$.

Lemma 3.1. Let $d=2\left\lceil n^{1 / 2}\right\rceil$ and $\mu=n^{1 / 2} / 2+3$. Let $\alpha$ be a fixed integer in the interval $[0, p-1]$. For any $\varepsilon>0$, sufficiently large $p$, and any element $g \in \mathbb{F}_{p}$ of multiplicative order $T \geq p^{1 / 3+\varepsilon}$ the following statement holds. Choose integers $x_{1}, \ldots, x_{d}$ uniformly and independently at random in the interval $[0, T-1]$. Then with probability $P \geq 1-2^{-n^{1 / 2}}$ for any vector $\mathbf{u}=\left(u_{1}, \ldots, u_{d}, 0\right)$ with

$$
\left(\sum_{i=1}^{d}\left(\left(\alpha g^{x_{i}} \operatorname{rem} p\right)-u_{i}\right)^{2}\right)^{1 / 2} \leq p 2^{-\mu}
$$

all vectors $\mathbf{v}=\left(v_{1}, \ldots, v_{d}, v_{d+1}\right) \in L_{g, p}\left(x_{1}, \ldots, x_{d}\right)$ satisfying

$$
\left(\sum_{i=1}^{d}\left(v_{i}-u_{i}\right)^{2}\right)^{1 / 2} \leq p 2^{-\mu}
$$

are of the form

with some $\beta \equiv \alpha(\bmod p)$.

$$
\mathbf{v}=\left(\left(\beta g^{x_{1}} \operatorname{rem} p\right), \ldots,\left(\beta g^{x_{d}} \operatorname{rem} p\right), \beta / p\right)
$$

Proof. As in [2] we define the modular distance between two integers $\beta$ and $\gamma$ as

$$
\operatorname{dist}_{p}(\beta, \gamma)=\min _{b \in \mathbb{Z}}|\beta-\gamma-b p|=\min \{((\beta-\gamma) \operatorname{rem} p), p-((\beta-\gamma) \operatorname{rem} p)\} .
$$

Let $x$ be an integer chosen uniformly at random in the interval $[0, T-1]$. It follows from Lemma 2.1 that for any $\beta$ and $\gamma$ with $\beta \not \equiv \gamma(\bmod p)$ the probability $P(\beta, \gamma)$ of

$$
\operatorname{dist}_{p}\left(\beta g^{x}, \gamma g^{x}\right)>p 2^{-\mu+1}
$$

for an integer $x$ chosen uniformly at random in the interval $[0, T-1]$ is

$$
P(\beta, \gamma)=1-2^{-\mu+2}+O\left(T^{-\delta}\right)
$$


for some $\delta>0$, depending only on $\varepsilon$. Hence

$$
P(\beta, \gamma) \geq 1-\frac{5}{2^{\mu}}
$$

provided that $p$ is large enough.

Therefore, for any $\beta \not \equiv \alpha(\bmod p)$,

$\operatorname{Pr}\left[\exists i \in[1, d] \mid \operatorname{dist}_{p}\left(\beta g^{x_{i}}, \alpha g^{x_{i}}\right)>p 2^{-\mu+1}\right]=1-(1-P(\alpha, \beta))^{d} \geq 1-\left(\frac{5}{2^{\mu}}\right)^{d}$,

where probability is taken over integers $x_{1}, \ldots, x_{d}$ chosen uniformly and independently at random in the interval $[0, T-1]$.

Since for $\beta \not \equiv \alpha(\bmod p)$ there are only $p-1$ possible values for $(\beta \operatorname{rem} p)$, we obtain

$$
\begin{aligned}
\operatorname{Pr}\left[\exists \beta \not \equiv \alpha \quad(\bmod p), \exists i \in[1, d] \mid \operatorname{dist}_{p}\left(\beta g^{x_{i}}, \alpha g^{x_{i}}\right)>p 2^{-\mu+1}\right] & \\
& \geq 1-(p-1)\left(\frac{5}{2^{\mu}}\right)^{d}>1-2^{-n^{1 / 2}}
\end{aligned}
$$

because

$$
d(\mu-\log 5)>\left\lceil n^{1 / 2}\right\rceil n^{1 / 2}+2\left\lceil n^{1 / 2}\right\rceil(3-\log 5)>\log p+n^{1 / 2} .
$$

The rest of the proof is identical to the proof of Theorem 5 of [2], we outline it for the sake of completeness.

Let us fix some integers $x_{1}, \ldots, x_{d}$ with

$$
\min _{\beta \neq \alpha} \min _{(\bmod p)} \operatorname{dist}_{i \in[1, d]}\left(\beta g^{x_{i}}, \alpha g^{x_{i}}\right)>p 2^{-\mu+1} .
$$

Let $\mathbf{v}$ be a lattice point satisfying

$$
\left(\sum_{i=1}^{d}\left(v_{i}-u_{i}\right)^{2}\right)^{1 / 2} \leq p 2^{-\mu}
$$

Clearly, since $\mathbf{v} \in L_{g, p}\left(x_{1}, \ldots, x_{d}\right)$, there are integers $\beta, z_{1}, \ldots, z_{d}$ such that

$$
\mathbf{v}=\left(\beta t_{1}-z_{1} p, \ldots, \beta t_{d}-z_{d} p, \beta / p\right),
$$

where, as in (4), $t_{i}=\left(g^{x_{i}} \operatorname{rem} p\right), i=1, \ldots, d$.

If $\beta \equiv \alpha(\bmod p)$, then for all $i=1, \ldots, d$ we have $\beta t_{i}-z_{i} p=\left(\beta t_{i} \operatorname{rem} p\right)$, for otherwise there would be $j \in\{1, \ldots, d\}$ so that $\left|v_{j}-u_{j}\right|>p 2^{-\mu}$.

Now suppose that $\beta \not \equiv \alpha(\bmod p)$. In this case we have

$$
\begin{aligned}
\left(\sum_{i=1}^{d}\left(v_{i}-u_{i}\right)^{2}\right)^{1 / 2} & \geq \min _{i \in[1, d]} \operatorname{dist}_{p}\left(\beta t_{i}, u_{i}\right) \\
& \geq \min _{i \in[1, d]}\left(\operatorname{dist}_{p}\left(\beta t_{i}, \alpha t_{i}\right)-\operatorname{dist}_{p}\left(u_{i}, \alpha t_{i}\right)\right) \\
& >p 2^{-\mu+1}-p 2^{-\mu}=p 2^{-\mu}
\end{aligned}
$$


that contradicts to our assumption. As we have seen, the condition (5) holds with probability exceeding $1-2^{-n^{1 / 2}}$ and the result follows.

For an integer $k \geq 1$ we define $f_{k}(t)$ by the inequalities

$$
\left(f_{k}(t)-1\right) \frac{p}{2^{k}} \leq(t \operatorname{rem} p)<f_{k}(t) \frac{p}{2^{k}} .
$$

Thus, roughly speaking, $f_{k}(t)$ is the integer defined by the $k$ most significant bits of $(t \operatorname{rem} p)$.

Using Lemma 3.1 in the same way as Theorem 5 is used in the proof of Theorem 1 of [2] we obtain

Lemma 3.2. Let $d=2\left\lceil n^{1 / 2}\right\rceil$ and $k=\left\lceil n^{1 / 2}\right\rceil+\lceil\log n\rceil$. For any $\varepsilon>0$, sufficiently large $p$ and any element $g \in \mathbb{F}_{p}$ of multiplicative order $T \geq p^{1 / 3+\varepsilon}$, there exists a deterministic polynomial time algorithm $\mathcal{A}$ such that for any integer $\alpha \in[1, p-1]$ given $2 d$ integers

$$
t_{i}=\left(g^{x_{i}} \operatorname{rem} p\right) \quad \text { and } \quad s_{i}=f_{k}\left(\alpha t_{i}\right), \quad i=1 \ldots, d,
$$

its output satisfies

$$
\operatorname{Pr}_{x_{1}, \ldots, x_{d} \in[0, T-1]}\left[\mathcal{A}\left(t_{1}, \ldots, t_{d} ; s_{1}, \ldots, s_{d}\right)=\alpha\right] \geq 1-2^{-n^{1 / 2}}
$$

if $x_{1}, \ldots, x_{d}$ are chosen uniformly and independently at random in the interval $[0, T-1]$.

Proof. We follow the same arguments as in the proof Theorem 1 of [2] which we briefly outline here for the sake of completeness. We refer to the first $d$ vectors in the defining matrix of $L_{g, p}\left(x_{1}, \ldots, x_{d}\right)$ as $p$-vectors.

Let us consider the vector $\mathbf{r}=\left(r_{1}, \ldots, r_{d}, r_{d+1}\right)$ where

$$
r_{i}=s_{i} \frac{p}{2^{k}}, \quad i=1, \ldots, d, \quad \text { and } \quad r_{d+1}=0 .
$$

Multiplying the last row vector $\left(t_{1}, \ldots, t_{d}, 1 / p\right)$ of the matrix (4) by $\alpha$ and subtracting certain multiples of $p$-vectors, we obtain a lattice point

$$
\mathbf{u}_{\alpha}=\left(u_{1}, \ldots, u_{d}, \alpha / p\right) \in L_{g, p}\left(x_{1}, \ldots, x_{d}\right)
$$

such that

$$
\left|u_{i}-r_{i}\right|<p 2^{-k}, \quad i=1, \ldots, d .
$$

Therefore,

$$
\left(\sum_{i=1}^{d+1}\left(u_{i}-r_{i}\right)^{2}\right)^{1 / 2} \leq p(d+1)^{1 / 2} 2^{-k}
$$


Now we can use the Babai algorithm [1] to find in polynomial time a lattice vector $\mathbf{v}=\left(v_{1}, \ldots, v_{d}, v_{d+1}\right) \in L_{g, p}\left(x_{1}, \ldots, x_{d}\right)$ such that

$$
\begin{aligned}
& \left(\sum_{i=1}^{d}\left(v_{i}-r_{i}\right)^{2}\right)^{1 / 2} \\
& \quad \leq 2^{(d+1) / 4} \min \left\{\left(\sum_{i=1}^{d+1}\left(z_{i}-r_{i}\right)^{2}\right)^{1 / 2}, \quad \mathbf{z}=\left(z_{1}, \ldots, z_{d}, z_{d+1}\right) \in L\right\} \\
& \quad \leq 2^{(d+1) / 4} p(d+1)^{1 / 2} 2^{-k} \leq p 2^{-\mu},
\end{aligned}
$$

where $\mu=n^{1 / 2} / 2+3$, provided that $n$ is sufficiently large. We also have

$$
\left(\sum_{i=1}^{d}\left(u_{i}-r_{i}\right)^{2}\right)^{1 / 2} \leq p d^{1 / 2} 2^{-k} \leq p 2^{-\mu} .
$$

Applying Lemma 3.1 , we see that $\mathbf{v}=\mathbf{u}_{\alpha}$ with probability at least $1-2^{-n^{1 / 2}}$, and therefore, $\alpha$ can be recovered in polynomial time.

Accordingly, using Lemma 2.2 instead of Lemma 2.1, in a similar way we obtain that for almost all primes much smaller values of $T$ can be considered.

Lemma 3.3. Let $Q$ be a sufficiently large integer. For any $\varepsilon>0$ there exists $\delta>0$ such that for all primes $p \in[Q, 2 Q]$, except at most $Q^{5 / 6+\varepsilon}$ of them, and any element $g \in \mathbb{F}_{p}$ of multiplicative order $T \geq p^{\varepsilon}$ there exists a deterministic polynomial time algorithm $\mathcal{A}$ such that for any integer $\alpha \in[1, p-1]$ given $2 d$ integers

$$
t_{i}=\left(g^{x_{i}} \mathrm{rem} p\right) \quad \text { and } \quad s_{i}=f_{k}\left(\alpha t_{i}\right), \quad i=1 \ldots, d,
$$

its output satisfies

$$
\operatorname{Pr}_{x_{1}, \ldots, x_{d} \in[0, T-1]}\left[\mathcal{A}\left(t_{1}, \ldots, t_{d} ; s_{1}, \ldots, s_{d}\right)=\alpha\right] \geq 1-2^{-n^{1 / 2}}
$$

if $x_{1}, \ldots, x_{d}$ are chosen uniformly and independently at random in the interval $[0, T-1]$.

\section{Security of the Most Significant Bits of the Diffie-Hellman Key}

We are ready to prove the main results.

Let $g \in \mathbb{F}_{p}$ be multiplicative order $T$. probabilistic polynomial time algorithm which for any pair $(a, b) \in[0, T-1]^{2}$, For each integer $k$ define the oracle $\mathcal{O}_{k, g}$ as a 'black box' which, any pair $(x, y) \in[0, T-1]^{2}$, given the values of $X=\left(g^{x} \operatorname{rem} p\right)$ and $Y=\left(g^{y}\right.$ rem $\left.p\right)$ outputs the value of $f_{k}\left(g^{x y}\right)$.

Theorem 4.1. Let $k=\left\lceil n^{1 / 2}\right\rceil+\lceil\log n\rceil$. For any $\varepsilon>0$, sufficiently large $p$ and any element $g \in \mathbb{F}_{p}$ of multiplicative order $T \geq p^{1 / 3+\varepsilon}$, there exists a probabilistic polynomial time algorithm which for any pair $(a, b) \in[0, T-1]^{2}$, given the values 
of $A=\left(g^{a} \operatorname{rem} p\right)$ and $B=\left(g^{b} \operatorname{rem} p\right)$, makes $O\left(n^{1 / 2}\right)$ calls of the oracle $\mathcal{O}_{k, g}$ and computes $\left(g^{a b} \operatorname{rem} p\right)$ correctly with probability $1+O\left(2^{-n^{1 / 2}}\right)$.

Proof. Given a pair $(a, b) \in[0, T-1]^{2}$ let us select an integer $r \in[0, T-1]$ uniformly at random. We compute

$$
g_{r}=\left(B g^{r} \operatorname{rem} p\right)
$$

thus $g_{r} \equiv g^{b+r}(\bmod p)$.

The probability that $\operatorname{gcd}(b+r, T) \geq T p^{-1 / 3-\varepsilon / 3}$ is at most $\tau(T) T^{-1} p^{1 / 3+\varepsilon / 3}$ where $\tau(T)$ is the number of positive integer divisors of $T$. Indeed, for any divisor $D \mid T$ with $D \geq T p^{-1 / 3-\varepsilon / 3}$ there are at most $T / D \leq p^{1 / 3+\varepsilon / 3}$ values of $s \in[0, T-1]$ with $\operatorname{gcd}(s, T)=D$.

Using the bound $\tau(T)=O\left(T^{\varepsilon / 3}\right)$, see Theorem 5.2 of Chapter 1 of [13], we obtain that the probability of $\operatorname{gcd}(b+r, T) \geq T p^{-1 / 3-\varepsilon / 3}$ is at most

$$
O\left(T^{-1} p^{1 / 3+2 \varepsilon / 3}\right)=O\left(p^{-\varepsilon / 3}\right)=O\left(2^{-n^{1 / 2}}\right) .
$$

In the opposite case, when $\operatorname{gcd}(a+r, T) \leq T p^{-1 / 3-\varepsilon / 3}$, the multiplicative order of $g_{r}$ is

$$
T_{r}=\frac{T}{\operatorname{gcd}(b+r, T)} \geq p^{1 / 3+\varepsilon / 3} .
$$

Let $\alpha_{r} \equiv g^{a(b+r)}(\bmod p)$. Then

$$
f_{k}\left(\alpha_{r} g_{r}^{x}\right)=f_{k}\left(g_{r}^{(a+x)}\right)=f_{k}\left(g^{(a+x)(b+r)}\right) .
$$

Now we can use the oracle $\mathcal{O}_{k, g}$ with $\left(g^{x} A \operatorname{rem} p\right)$ and $\left(g^{r} B \operatorname{rem} p\right)$ to evaluate $f_{k}\left(\alpha_{r} g_{r}^{x}\right)$ for an integer $x$ chosen uniformly at random in the interval $[0, p-1]$. Because $T_{r} \mid p-1$ the values of $\left(x \operatorname{rem} T_{r}\right)$ are uniformly distributed in the interval $\left[0, T_{r}-1\right]$ as well, thus Lemma 3.2 can be applied. Therefore, one can construct a probabilistic polynomial time algorithm that:

- Selects a random $r \in[0, T-1]$.

- Applies algorithm $\mathcal{A}$ from Lemma 3.2 (now $g_{r}$ plays the role of $g$ in the conditions of Lemma 3.2). This algorithm makes $O\left(n^{1 / 2}\right)$ calls to the oracle $\mathcal{O}_{k, g}$.

- Outputs the correct value of $\alpha_{r}$ with probability at least $1-O\left(2^{-n^{1 / 2}}\right)$.

Indeed, the only possible source of error is either the case $T_{r} \leq p^{1 / 3+\varepsilon / 3}$ or the probability error of the algorithm of Lemma 3.2. The probability of both events is $O\left(2^{-n^{1 / 2}}\right)$.

Remarking that

$$
g^{a b} \equiv \alpha_{r} A^{-r} \quad(\bmod p),
$$

we obtain the desired result. 
It is easy to see that Theorem 4.1 is nontrivial for any $T \geq p^{1 / 3+\varepsilon}$. In a similar way, Lemma 2.2 produces a result which holds for almost all primes $p$ and is non-trivial for $T \geq p^{\varepsilon}$.

Theorem 4.2. Let $k=\left\lceil n^{1 / 2}\right\rceil+\lceil\log n\rceil$. For any $\varepsilon>0$ and for all primes $p \in$ $\left[2^{n-1}, 2^{n}-1\right]$, except at most $2^{(5 / 6+\varepsilon) n}$ of them, and any element $g \in \mathbb{F}_{p}$ of multiplicative order $T \geq p^{\varepsilon}$ the following statement holds: There exists a probabilistic polynomial time algorithm which for any pair $(a, b) \in[0, T-1]^{2}$, given the values of $A=\left(g^{a} \operatorname{rem} p\right)$ and $B=\left(g^{b} \operatorname{rem} p\right)$, makes $O\left(n^{1 / 2}\right)$ calls of the oracle $\mathcal{O}_{k, g}$ and computes $\left(g^{a b} \operatorname{rem} p\right)$ correctly with probability $1+O\left(2^{-n^{1 / 2}}\right)$.

\section{Remarks}

First of all we note that the constants in above estimates are effective and can be explicitly evaluated.

It would be very interesting to replace the condition $T \geq p^{\varepsilon}$ for the smallest size of the multiplicative order of $g$ in Lemma 2.2 by a weaker condition of the form $T \geq(\log p)^{c}$ with some constant $c$. Although a more careful analysis of the proof of Theorem 5.5 of [7] should allow to replace $p^{\varepsilon}$ with a slower growing function, it seems unlikely that the present method can be applied to $T$ as small as a power of $\log p$.

Our results can also be applied to several other cryptosystems based on exponentiation in finite fields, which have been considered in [2], except the Shamir message passing scheme, see [2, 3] (this scheme is also described in Protocol 12.22 in [11]). Unfortunately the proof of Theorem 3 in [2] suffers from the same problem as the proof of Theorem 2 of that paper. Namely, for the ElGamal scheme, see $[2,3]$ as well as Section 8.4 from [11], it produces a result which applies only to at most $50 \%$ of the cases and it cannot be applied to the the Shamir message passing scheme at all. Indeed, in this scheme the exponent $x$ of the corresponding multiplier $g^{x}$ must satisfy the additional condition $\operatorname{gcd}(b x+1, p-1)=1$, with some $b, \operatorname{gcd}(b, p-1)=1$, thus $g^{x}$ runs through some special subset of $\mathbb{F}_{p}^{*}$ (even if $g$ is a primitive root) rather than through the whole $\mathbb{F}_{p}^{*}$ and thus Theorem 1 of $[2]$ does not apply. Our results in their present form cannot be used for this problem directly, however it has been shown in [6] that a modification of the technique of this paper, combined with some elementary sieve method produce similar results for the Shamir message passing scheme.

Besides the mentioned in $[2,3]$ cryptosystems several other schemes can be studied as well. For example, very similar results hold for the MatsumotoTakachima-Imai key-agreement protocol, see Section 12.6 of [11].

The results of [3] can be generalized in a similar way. To do so one can use the bound of exponential sums of Theorem 3.4 of [7] to study the distribution of the sums $\left(g^{x_{1}}+\ldots+g^{x_{r}} \operatorname{rem} p\right)$ and thus obtain an analogue of Lemma 2.4 of [3]. 
One can also extend Theorem 4.1 to the case of Diffie-Hellman encryption modulo an arbitrary composite integer $m \geq 2$. Indeed, using the well-known bound

$$
\max _{\operatorname{gcd}(c, m)=1}\left|\sum_{x=0}^{T-1} \exp \left(2 \pi i c g^{x} / m\right)\right| \leq m^{1 / 2},
$$

see Theorem 10 of Chapter 1 in [8] or Theorem 8.2 in [12], instead of (1) and (2), one can obtain similar results for elements $g, \operatorname{gcd}(g, m)=1$, of multiplicative order $T$ modulo $m$ such that $T \geq m^{1 / 2+\varepsilon}$. In fact, Lemma 3.2 can be extended to elements $t_{i}$ chosen uniformly and independently at random from any subgroup $\mathcal{G}$ of the group of units modulo $m$, provided that the cardinality of $\mathcal{G}$ satisfies $\# \mathcal{G} \geq m^{1 / 2+\varepsilon}$

As we have mentioned, similar but somewhat more involved technique can be applied to studying the bit security of the Shamir message passing scheme, see [6].

Finally, we remark that somewhat similar problem for extensions of finite fields have been considered in [16]. The results of that paper and some of their improvements in [15] have applications to the security of the new cryptosystem designed in $[4,10]$.

Acknowledgement. We thank Consuelo Martínez for her interest and for helpful advice. We also thank Mats Näslund for a careful reading of the manuscript and fruitful discussion.

\section{References}

[1] L. Babai, On Lovász' lattice reduction and the nearest lattice point problem, Combinatorica, 6 (1986), 11-13.

[2] D. Boneh and R. Venkatesan, Hardness of computing the most significant bits of secret keys in Diffie-Hellman and related schemes, Lect. Notes in Comp. Sci., SpringerVerlag, Berlin, 1109 (1996), 129-142.

[3] D. Boneh and R. Venkatesan, Rounding in lattices and its cryptographic applications, Proc. 8-rd Annual ACM-SIAM Symp. on Discr. Algorithms, ACM, NY, 1997, 675681

[4] A. E. Brouwer, R. Pellikan, and E. R. Verheul, Doing more with fewer bits, Lect. Notes in Comp. Sci., Springer-Verlag, Berlin, 1716, (1999), 321-332.

[5] M. I. González Vasco and M. Näslund, A survey of hard core functions, Preprint, 2000.

[6] M. I. González Vasco and I. E. Shparlinski, Security of the most significant bits of the Shamir message passing scheme, Preprint, 2000, 1-14.

[7] S. V. Konyagin and I. E. Shparlinski, Character sums with exponential functions and their applications, Cambridge Univ. Press, Cambridge, 1999.

[8] N. M. Korobov, Exponential sums and their applications, Kluwer Acad. Publ., Dordrecht, 1992.

[9] A. K. Lenstra, H. W. Lenstra and L. Lovász, Factoring polynomials with rational coefficients, Mathematische Annalen, 261 (1982), 515-534. 
[10] A. K. Lenstra and E. R. Verheul, The XTR public key system, Proc. of Crypto'2000, Springer-Verlag, Berlin, (to appear).

[11] A. J. Menezes, P. C. van Oorschot and S. A. Vanstone, Handbook of Applied Cryptography, CRC Press, Boca Raton, FL, 1996.

[12] H. Niederreiter, Quasi-Monte Carlo methods and pseudo-random numbers, Bull. Amer. Math. Soc., 84 (1978), 957-1041.

[13] K. Prachar, Primzahlverteilung, Springer-Verlag, Berlin, 1957.

[14] C. P. Schnorr, A hierarchy of polynomial time basis reduction algorithms, Theor. Comp. Sci., 53 (1987) 201-224.

[15] I. E. Shparlinski, Security of polynomial transformations of the Diffie-Hellman key, Preprint, 2000.

[16] E. R. Verheul, Certificates of recoverability with scalable recovery agent security, Lect. Notes in Comp. Sci., Springer-Verlag, Berlin, 1751 (2000), 258-275.

[17] I. M. Vinogradov, Elements of number theory, Dover Publ., New York, 1954.

Department of Mathematics, University of Oviedo, Oviedo, 33007, Spain

E-mail address: mvasco@orion.ciencias.uniovi.es

Department of Computing, Macquarie University, Sydney, NSW 2109, Australia

E-mail address: igor@comp.mq.edu.au 